Kragujevac Journal of Mathematics

Volume 39(2) (2015), Pages 149-154.

\title{
NORMAL FAMILIES OF MEROMORPHIC FUNCTIONS CONCERNING SHARED FUNCTIONS
}

\author{
CHENG-XIONG SUN ${ }^{1}$
}

\begin{abstract}
It is mainly proved: Let $\mathfrak{F}$ be a family of meromorphic function in $\mathcal{D}$, $a(z)(\neq 0)$ and $b(z)(\neq 0)$ be two holomorphic functions on $\mathcal{D}$. Suppose that admits the zeros of multiplicity at least 3 for each function $f \in \mathfrak{F}$. For each $f \in \mathfrak{F}$, if $f=a(z) \Leftrightarrow f^{\prime}=b(z)$, then $\mathfrak{F}$ is normal in $\mathcal{D}$. Some example shows that the multiplicity of zeros of $f$ is best in some sense. And the result of paper improve and supplement the result of Lei, Yang and Fang [J. Math. Anal. App. 364 (2010), 143-150].
\end{abstract}

\section{Introduction And Main Results}

Let $\mathcal{D}$ be a domain in $\mathbb{C}$, and $\mathfrak{F}$ be a family of meromorphic functions defined in the domain $\mathcal{D} . \mathfrak{F}$ is said to be normal in $\mathcal{D}$, in the sense of Montel, if for every sequence $\left\{f_{n}\right\}_{n=1}^{\infty}$ contains a subsequence $\left\{f_{n_{j}}\right\}_{j=1}^{\infty}$ such that $f_{n_{j}}$ converges spherically uniformly to a meromorphic function $f(z)$ or $\infty$ (see [1]).

A family $\mathfrak{F}$ is said to be normal at a point $z_{0} \in \mathcal{D}$ if there exists a neighborhood of $z_{0}$ in which $\mathfrak{F}$ is normal. It is well known that $\mathfrak{F}$ is normal in a domain $\mathcal{D}$ if and only if it is normal at each of its points (see [1]).

Let $f(z)$ and $g(z)$ be two meromorphic functions in $\mathcal{D}$ and $a, b \in \mathbb{C}$. If $g(z)=b$ whenever $f(z)=a$, we write $f(z)=a \Rightarrow g(z)=b$. If $f(z)=a \Rightarrow g(z)=b$ and $g(z)=b \Rightarrow f(z)=a$, we write $f(z)=a \Leftrightarrow g(z)=b$.

In 2002, Fang and Zalcman [2] proved the following theorem.

Theorem 1.1. Let $\mathfrak{F}$ be a family of meromorphic functions in a domain $\mathcal{D}$ and $a, b$ be two nonzero complex numbers. Let $k$ be a positive integer. Suppose that admits

Key words and phrases. Meromorphic functions, holomorphic functions, normal family, shared functions.

2010 Mathematics Subject Classification. Primary: 30D45.

Received: October 8, 2013.

Revised: June 25, 2015. 
the zeros of multiplicity at least $k+1$ for each function $f \in \mathfrak{F}$. For each $f \in \mathfrak{F}$, if $f=a \Leftrightarrow f^{(k)}=b$, then $\mathfrak{F}$ is normal in $\mathcal{D}$.

In 2010, Lei, Yang and Fang [3] extended the constants $a, b$ in Theorem 1.1 to holomorphic functions $a(z)(\neq 0), b(z)(\not \equiv 0)$, as follows.

Theorem 1.2. [4] Let $\mathfrak{F}$ be a family of meromorphic functions in a domain $\mathcal{D}$ and $a(z)(\neq 0), b(z)(\neq \equiv 0)$ be two holomorphic functions. Let $k(\geq 2)$ be a positive integer. Suppose that admits the zeros of multiplicity at least $k+1$ for each function $f \in \mathfrak{F}$. For each $f \in \mathfrak{F}$, if $f=a(z) \Leftrightarrow f^{(k)}=b(z)$, then $\mathfrak{F}$ is normal in $\mathcal{D}$.

Naturally, we pose the following question: Is the conclusion of Theorem 1.2 also true for $k=1$.

First, we give the following counterexample.

Example 1.1. Let $\mathcal{D}=\{z:|z|<1\}, a(z)=\frac{z^{2}+2}{2}, b(z)=z$. Let $\mathfrak{F}=\left\{f_{n}\right\}$ where $f_{n}(z)=\frac{\left(z^{2}-\frac{1}{n}\right)^{2}}{2\left(z^{2}+\frac{1}{2 n^{2}}\right)}, z \in \mathcal{D}(n=1,2, \ldots)$. Clearly, all the zeros of $f_{n}(z)$ are multiple, and $f_{n}(z)-a(z)=-\frac{\left(1+\frac{1}{2 n}\right)^{2} z^{2}}{z^{2}+\frac{1}{2 n^{2}}}, f_{n}^{\prime}(z)-b(z)=-\frac{\left(1+\frac{1}{2 n}\right)^{2} z}{n^{2}\left(z^{2}+\frac{1}{2 n^{2}}\right)^{2}}$. Thus $f_{n}(z)-a(z) \neq 0$, $f_{n}^{\prime}(z)-b(z) \neq 0$ in $\mathbb{C} \backslash\{0\}$. It follows that $f_{n}(z)-a(z)=0 \Leftrightarrow f_{n}^{\prime}(z)-b(z)=0$, this is $f_{n}(z)=a(z) \Leftrightarrow f_{n}^{\prime}(z)=b(z)$, however, $\mathfrak{F}$ fails to be normal in $\mathcal{D}$ since $f_{n}\left(\frac{1}{\sqrt{n}}\right)=0, f_{n}\left(\frac{1}{\sqrt{2} i n}\right)=\infty$ as $n \rightarrow \infty$.

Example 1.1 shows that the conclusion of Theorem 1.2 does not hold for $k=1$. This suggests that some further investigation is necessary for the case $k=1$. In the paper we take up this problem and prove the following result.

Theorem 1.3. Let $\mathfrak{F}$ be a family of meromorphic function in $\mathcal{D}, a(z)(\neq 0)$ and $b(z)(\not \equiv 0)$ be two holomorphic functions on $\mathcal{D}$. Suppose that admits the zeros of multiplicity at least 3 for each function $f \in \mathfrak{F}$. For each $f \in \mathcal{F}$, if $f=a(z) \Leftrightarrow f^{\prime}=$ $b(z)$, then $\mathfrak{F}$ is normal in $\mathcal{D}$.

Example 1.2. Let $\mathcal{D}=\{z:|z|<1\}, a(z)=\frac{z^{2}}{2}, b(z)=z$. Let $\mathfrak{F}=\left\{f_{n}\right\}$ where $f_{n}=\frac{z^{4}}{2\left(z^{2}-\frac{1}{n}\right)}, z \in D(n=1,2, \ldots)$. Then $f_{n}(z)-\frac{1}{2} z^{2}=\frac{\frac{z^{2}}{n}}{2\left(z^{2}-\frac{1}{n}\right)}, f_{n}^{\prime}(z)-z=-\frac{\frac{z}{n^{2}}}{\left(z^{2}-\frac{1}{n}\right)^{2}}$. Clearly, $f_{n}(z)=a(z) \Leftrightarrow f_{n}^{\prime}(z)=b(z)$, however, $\mathfrak{F}$ fails to be normal in $\mathcal{D}$ since $f_{n}\left(\frac{1}{\sqrt{n}}\right)=\infty, f_{n}(0)=0$ as $n \rightarrow \infty$.

Remark 1.1. Example 1.1 shows that the condition that all zeros of $f$ have multiplicity at least 3 in Theorems 1.3 is shape. Example 1.2 shows that the condition that $a(z) \neq 0$ is necessary. 


\section{Auxiliary Results}

To prove our result, we require some preliminary results.

Lemma 2.1. [4] Let $\mathfrak{F}$ be a family of functions meromorphic on a domain $\mathcal{D}$, all of whose zeros have multiplicity at least $k$. Suppose that there exists $A \geqslant 1$ such that $\left|f^{(k)}(z)\right| \leqslant A$ whenever $f(z)=0$. Then if $\mathfrak{F}$ is not normal at $z_{0} \in \mathcal{D}$, there exist, for each $0 \leqslant \alpha \leqslant k$,

(i) points $z_{n}, z_{n} \rightarrow z_{0}$;

(ii) functions $f_{n} \in \mathfrak{F}$; and

(iii) positive numbers $\rho_{n} \rightarrow 0^{+}$

such that $\rho_{n}^{-\alpha} f_{n}\left(z_{n}+\rho_{n} \xi\right)=g_{n}(\xi) \rightarrow g(\xi)$ locally uniformly with respect to the spherical metric, where $g(\xi)$ is a nonconstant meromorphic function on $\mathbb{C}$, all of whose zeros of $g(\xi)$ are of multiplicity at least $k$, and order at most 2.

Lemma 2.2. [5] Let $f(z)$ be a transcendental meromorphic function with finite order, all of whose zeros are of multiplicity at least 2 , and let $P(z)(\not \equiv 0)$ be a polynomial, then $f^{\prime}(z)-P(z)$ has infinitely many zeros.

Lemma 2.3. [5] Let $k$ be a positive integer, $f(z)$ be a meromorphic function with finite order, all of whose zeros have multiplicity at least $k+2$. If $f^{(k)} \neq 1$, then $f(z)$ is a constant function.

Lemma 2.4. [6] Let $k, l$ be positive integers, $Q(z)$ be a non-constant rational function, all of whose zeros have multiplicity at least $k+2$. If $Q^{(k)}(z) \neq z^{l}$, then $l=1$ and $Q^{(k)}(z)=\frac{1}{(k+1) !} \frac{(z+c)^{k+2}}{(z+(k+2) c)}$, where $c$ is a nonzero constant.

Lemma 2.5. Let $\left\{f_{n}\right\}$ be a family of meromorphic functions in a domain $\mathcal{D}$, all of whose zeros are of multiplicity at least 3 , and let $a_{n}(z), b_{n}(z)$ be two sequences of analytic functions in $\mathcal{D}$ such that $a_{n}(z) \rightarrow a(z) \neq 0, b_{n}(z) \rightarrow b(z)$. If $f_{n}^{\prime}(z)=b_{n}(z) \Rightarrow$ $f_{n}(z)=a_{n}(z)$, then $\left\{f_{n}\right\}$ is normal in $\mathcal{D}$.

Proof. Suppose that $\left\{f_{n}\right\}$ is not normal at $z_{0} \in \mathcal{D}$. We may assume that $b\left(z_{0}\right)=1$. By Lemma 2.1, there exists a sequence of complex numbers $z_{n} \rightarrow z_{0}$, a sequence of functions $f_{n} \in\left\{f_{n}\right\}$ and a sequence of positive numbers $\rho_{n} \rightarrow 0$ such that $\rho_{n}^{-1} f_{n}\left(z_{n}+\right.$ $\left.\rho_{n} \xi\right)=g_{n}(\xi) \rightarrow g(\xi)$ locally uniformly with respect to the spherical metric to a nonconstant meromorphic functions $g(\xi)$ on $\mathbb{C}$. Also the order of $g(\xi)$ does not exceed 2 and $g(\xi)$ has no zero of multiplicity less than 3 .

We claim $g^{\prime}(\xi) \neq 1$.

If this is not true, then $g^{\prime}\left(\xi_{0}\right)=1$. So we have $g^{\prime}(\xi) \not \equiv 1$, otherwise $g(\xi)$ must be a polynomial with $\operatorname{deg}(g)=1$, which contradicts the fact that each zero of $g(\xi)$ has multiplicity at least 3 . Since $g^{\prime}\left(\xi_{0}\right)=1=b\left(\xi_{0}\right)$, then there exist $\xi_{n} \rightarrow \xi_{0}$ such that (for $n$ sufficiently large) $f_{n}^{\prime}\left(z_{n}+\rho_{n} \xi_{n}\right)=g_{n}^{\prime}\left(\xi_{n}\right)=b\left(z_{n}+\rho_{n} \xi_{n}\right)$. It follows that $f_{n}\left(z_{n}+\rho_{n} \xi_{n}\right)=a\left(z_{n}+\rho_{n} \xi_{n}\right)$, then $g_{n}\left(\xi_{n}\right)=\rho_{n}^{-1} f_{n}\left(z_{n}+\rho_{n} \xi\right)=\rho_{n}^{-1} a_{n}\left(z_{n}+\rho_{n} \xi_{n}\right)$. Thus $g\left(\xi_{0}\right)=\infty$, which contradicts $g^{\prime}\left(\xi_{0}\right)=1$. This proves $g^{\prime}(\xi) \neq 1$. By Lemma 2.3 $g(\xi)$ is a constant, a contradiction. Thus $\left\{f_{n}\right\}$ is normal in $\mathcal{D}$. 


\section{Proof of TheOREM 1.3}

Proof. For any point $z_{0} \in \mathcal{D}$, either $b\left(z_{0}\right)=0$ or $b\left(z_{0}\right) \neq 0$. We consider two cases.

Case $1 . b\left(z_{0}\right) \neq 0$. Then by Lemma 2.5 , we get that $\mathfrak{F}$ is normal at $z_{0}$.

Case 2. $b\left(z_{0}\right)=0$. Let $z_{0}=0, \mathcal{D}=\Delta=\{z:|z|<1\}$ and $b(z)=z^{m}+a_{m+1} z^{m+1}+$ $\cdots=z^{m} \phi(z), \phi(0)=1, \phi(z) \neq 1, z \in\{z: 0<|z|<1\}$.

Suppose that $\mathfrak{F}$ is not normal in $\mathcal{D}$. Without loss of generality, we assume that $\mathfrak{F}$ is not normal at $z_{0}=0$.

Consider the family as follows

$$
\mathfrak{G}=\left\{g(z)=\frac{f(z)}{z^{m}}, f \in \mathfrak{F}\right\} .
$$

Then $\mathfrak{G}$ is not normal at $z_{0}=0$ in $\mathcal{D}$. Applying Lemma 2.1, there exists a sequence of complex numbers $z_{n} \rightarrow z_{0}$, a sequence of functions $f_{n} \in\left\{f_{n}\right\}$ and a sequence of positive numbers $\rho_{n} \rightarrow 0$ such that

$$
G_{n}(\xi)=\frac{g_{n}\left(z_{n}+\rho_{n} \xi\right)}{\rho_{n}}=\frac{f_{n}\left(z_{n}+\rho_{n} \xi\right)}{\rho_{n}\left(z_{n}+\rho_{n} \xi\right)^{m}} \rightarrow G(\xi)
$$

locally uniformly with respect to the spherical metric to a non-constant meromorphic functions $G(\xi)$ on $\mathbb{C}$. Also the order of $G(\xi)$ does not exceed 2 and $G(\xi)$ has no zero of multiplicity less than 3 .

Next, we consider two cases.

Case 2.1. We may suppose that $\frac{z_{n}}{\rho_{n}} \rightarrow \infty$. From (3.1), we have

$$
\widetilde{G}_{n}(\xi):=\frac{f_{n}\left(z_{n}+\rho_{n} \xi\right)}{\rho_{n} z_{n}^{m}}=\left(1+\frac{\rho_{n}}{z_{n}}\right)^{m} G_{n}(\xi) \rightarrow G(\xi)
$$

on $\mathbb{C}$, then we have $\widetilde{G}_{n}^{\prime} \rightarrow G^{\prime}$ on $\mathbb{C} \backslash G^{-1}(\infty)$. We claim $G^{\prime} \neq 1$ on $\mathbb{C}$.

Suppose that $G^{\prime}\left(\xi_{0}\right)=1$ for $\xi_{0} \in \mathbb{C}$. Then we get $G^{\prime} \not \equiv 1$, otherwise $G(\xi)=\xi+c$, where $c$ is a non-zero constant, which contradicts the fact each zero of $G(\xi)$ has multiplicity at least 3 .

Since $\widetilde{G}_{n}^{\prime}(\xi)-\left(1+\frac{\rho_{n}}{z_{n}} \xi\right)^{m} \phi\left(z_{n}+\rho_{n} \xi\right) \rightarrow G^{\prime}(\xi)-1$ on $\mathbb{C} \backslash G^{-1}(\infty)$, then there exists $\xi_{n} \rightarrow \xi_{0}$, such that $\widetilde{G}_{n}^{\prime}\left(\xi_{n}\right)-\left(1+\frac{\rho_{n}}{z_{n}} \xi_{n}\right)^{m} \phi\left(z_{n}+\rho_{n} \xi_{n}\right)=0$. Thus, for $n$ sufficiently large, we obtain

$$
f_{n}^{\prime}\left(z_{n}+\rho_{n} \xi_{n}\right)=z_{n}^{m} \widetilde{G}_{n}^{\prime}\left(\xi_{n}\right)=z_{n}^{m}\left(1+\frac{\rho_{n}}{z_{n}} \xi_{n}\right)^{m} \phi\left(z_{n}+\rho_{n} \xi_{n}\right)=b\left(z_{n}+\rho_{n} \xi_{n}\right) .
$$

It follows that $f_{n}\left(z_{n}+\rho_{n} \xi_{n}\right)=a\left(z_{n}+\rho_{n} \xi_{n}\right)$. From (3.2) we have

$$
G\left(\xi_{0}\right)=\lim _{n \rightarrow \infty} \widetilde{G}_{n}\left(\xi_{n}\right)=\lim _{n \rightarrow \infty} \frac{f_{n}\left(z_{n}+\rho_{n} \xi\right)}{\rho_{n} z_{n}^{m}}=\lim _{n \rightarrow \infty} \frac{a\left(z_{n}+\rho_{n} \xi\right)}{\rho_{n} z_{n}^{m}}=\infty,
$$


which contradicts $G^{\prime}\left(\xi_{0}\right)=1$. So $G^{\prime}(\xi) \neq 1$. By Lemma $2.3 G$ is a constant, a contradiction.

Case 2.2. We may suppose that $\frac{z_{n}}{\rho_{n}} \rightarrow \alpha$, a finite complex number. From (3.1), we have

$$
\widehat{G}_{n}(\xi):=\frac{f_{n}\left(\rho_{n} \xi\right)}{\rho_{n}^{m+1} \xi^{m}}=G_{n}\left(\xi-\frac{z_{n}}{\rho_{n}}\right) \rightarrow G(\xi-\alpha):=\widehat{G}(\xi)
$$

on $\mathbb{C}$. Then $\widehat{G}(\xi)$ has no a zero of multiplicity less than 3 , and the pole of $\widehat{G}(\xi)$ at $\xi=0$ has multiplicity at least $m$.

Now set $F_{n}(\xi):=\frac{f_{n}\left(\rho_{n} \xi\right)}{\rho_{n}^{m+1}}$, and $F(\xi)=\xi^{m} \widehat{G}(\xi)$. From $(3.3)$, we can get $F_{n}(\xi)=$ $\xi^{m} \widehat{G}_{n}(\xi) \rightarrow F(\xi)$. Clearly $F_{n}(\xi) \rightarrow F(\xi)$ on $\mathbb{C}, F(0) \neq 0$ and the zero of $F(\xi)$ has multiplicity at least 3 .

We claim: (i) $F^{\prime}(\xi) \neq \xi^{m}$, (ii) all the poles of $F(\xi)$ are multiple.

First, we now prove that $F^{\prime}(\xi) \not \equiv \xi^{m}$. Otherwise $F(\xi)=\frac{1}{m+1} \xi^{m+1}+d$, where $d$ is a constant. Since $F(0) \neq 0$, then $d \neq 0$. So $F(\xi)$ has only simple zeros, contradiction. Thus let $\xi_{0} \in \mathbb{C}$ with $F^{\prime}\left(\xi_{0}\right)=\xi_{0}{ }^{m}$, then $F(\xi)$ is holomorphic at $\xi_{0}$. Therefore, we can obtain

$$
\frac{f^{\prime}{ }_{n}\left(\rho_{n} \xi\right)-b\left(\rho_{n} \xi\right)}{\rho_{n}^{m}}=F_{n}^{\prime}(\xi)-\xi^{m} \phi\left(\rho_{n} \xi\right) \rightarrow F^{\prime}(\xi)-\xi^{m} .
$$

By Hurwitz's theorem, there exists point $\xi_{n} \rightarrow \xi_{0}$ such that $f_{n}^{\prime}\left(\rho_{n} \xi_{n}\right)-b\left(\rho_{n} \xi_{n}\right)=0$ for $n$ sufficiently large. So we have $f_{n}\left(\rho_{n} \xi_{n}\right)-a\left(\rho_{n} \xi_{n}\right)=0$, this is $f_{n}\left(\rho_{n} \xi_{n}\right)=a\left(\rho_{n} \xi_{n}\right)$. Noting that $a(0) \neq 0$, thus

$$
F\left(\xi_{0}\right)=\lim _{n \rightarrow \infty} F_{n}\left(\xi_{n}\right)=\lim _{n \rightarrow \infty} \frac{f_{n}\left(\rho_{n} \xi_{n}\right)}{\rho_{n}^{m+1}}=\lim _{n \rightarrow \infty} \frac{a\left(\rho_{n} \xi_{n}\right)}{\rho_{n}^{m+1}}=\infty,
$$

which contradicts that $F(\xi)$ is holomorphic at $\xi_{0}$. This proves (i).

Next we prove (ii). Suppose $F\left(\xi_{0}\right)=\infty$. There exists a $\bar{\Delta}=\left\{\xi:\left|\xi-\xi_{0}\right| \leq \delta\right\}$ such that $\frac{1}{F(\xi)}$ is holomorphic and $\xi_{0}$ is the zero of $\frac{1}{F(\xi)}$. Hence $\frac{1}{F_{n}(\xi)}-\frac{\rho_{n}^{m+1}}{a\left(\rho_{n} \xi\right)} \rightarrow \frac{1}{F(\xi)}$ on $\bar{\Delta}$ and $\frac{1}{F(\xi)} \not \equiv 0$. It follows that there exists $\xi_{n} \rightarrow \xi_{0}$ such that $\frac{1}{F_{n}\left(\xi_{n}\right)}-\frac{\rho_{n}^{m+1}}{a\left(\rho_{n} \xi_{n}\right)}=0$ for $n$ sufficiently large. Therefore $f_{n}^{\prime}\left(\rho_{n} \xi_{n}\right)=b\left(\rho_{n} \xi_{n}\right)$. So we get

$$
F_{n}^{\prime}\left(\xi_{n}\right)=\frac{f_{n}^{\prime}\left(\rho_{n} \xi_{n}\right)}{\rho_{n}^{m}}=\frac{b\left(\rho_{n} \xi_{n}\right)}{\rho_{n}^{m}}=\phi\left(\rho_{n} \xi_{n}\right) \xi_{n}^{m} \rightarrow \xi_{0}^{m} .
$$

Thus we have $\left.\left(\frac{1}{F_{n}(\xi)}\right)^{\prime}\right|_{\xi=\xi_{0}}=-\left.\frac{F_{n}^{\prime}(\xi)}{F_{n}^{2}(\xi)}\right|_{\xi=\xi_{0}}=0$ and $\left(\frac{1}{F_{n}(\xi)}\right)^{\prime} \rightarrow\left(\frac{1}{F(\xi)}\right)^{\prime}$. It follows that $\xi_{0}$ is a multiple zero of $\left(\frac{1}{F(\xi)}\right)^{\prime}$, this is, $\xi_{0}$ is a multiple pole of $F(\xi)$. This proves (ii).

By Lemma 2.2, $F(\xi)$ must be a rational function. Then by Lemma 2.4, we have $m=1$ and $F(\xi)=\frac{1}{2} \frac{(\xi+c)^{3}}{(\xi+3 c)}$, which contradicts the fact $F(\xi)$ has multiple poles. Hence we show that $\mathfrak{G}$ is normal at $z_{0}=0$. 
Next, we show that $\mathfrak{F}$ is normal at $z_{0}=0$. Since $\mathfrak{G}$ is normal at $z_{0}=0$, let $g_{n} \rightarrow g$ in a neighborhood of 0 , then there exist $\Delta_{\delta}=\{z:|z|<\delta\}$ and a subsequence of $\left\{g_{n}\right\}$ such that $\left\{g_{n}\right\}$ converges uniformly to a meromorphic function or $\infty$. Noting $g(0)=\infty$, we can find a $\varepsilon$ with $0<\varepsilon<\delta$ and $M>0$ such that $|g(z)|>M, z \in \Delta_{\varepsilon}$. So, for sufficiently large $n$, we get $\left|g_{n}(z)\right| \geq \frac{M}{2}$. Therefore $f_{n}(z) \neq 0$ for sufficiently large $n$ and $z \in \Delta_{\varepsilon}$.

Hence $\frac{1}{f_{n}}$ is analytic in $\Delta_{\varepsilon}$. It follows that, for sufficiently large $n$,

$$
\left|\frac{1}{f_{n}(z)}\right|=\left|\frac{1}{g_{n}(z)}\right| \frac{1}{|z|^{m}} \leq\left(\frac{2}{\varepsilon}\right)^{m} \frac{2}{M}, \quad|z|=\frac{\varepsilon}{2} .
$$

By the Maximum Principle and Montel's theorem, $\mathfrak{F}$ is normal at $z_{0}=0$.

These shows that $\mathfrak{F}$ is normal in $\mathcal{D}$.

Acknowledgments. The author would like to thank the referee for several helpful suggestions toward this paper.

\section{REFERENCES}

[1] J. Schiff, Normal Families, Springer-Verlag, Berlin, 1993.

[2] M. L. Fang and L. Zalcman, Normal families and shared values III, Comput. Methods Funct. Theory 1 (2002), 385-395.

[3] C. L. Lei and D. G. Yang, M. L. Fang, Normal families and shared values of meromorphic functions, J. Math. Anal. Appl. 364 (2010), 143-150.

[4] L. Zalcman, Normal families: new perspectives, Bull. Amer. Math. Soc. (N.S.) 35(3) (1998), 215-230.

[5] Y. F. Wang and M. L. Fang, Picard values and normal families of meromorphic functions with multiple zeros, Acta Math. Sinica (N.S.) 14(5) (1998), 17-26.

[6] C. N. Chen and Y. Xu, Normal families of meromorphic functions concerning differential polynomials, Houston J. Math. 39(2) (2013), 611-623.

${ }^{1}$ Department of Mathematics,

Xuanwei Senior School,

Yunnan Xuanwei 655400,PR China

E-mail address: ynxwjz@hotmail.com 